\title{
Pretreatment MRI in Primary Rectal Cancer as a Predictor for Oncological Outcomes After Surgery for Local Recurrence
}

\author{
ANDERS H. ELLIOT ${ }^{1}$, BARWAR N. OTHMAN ${ }^{2}$, CHIKAKO SUZUKI ${ }^{3}$, LENNART BLOMQVIST $^{3}$, \\ ANNA MARTLING ${ }^{1}$, HEMMING JOHANSSON ${ }^{4}$, BENGT GLIMELIUS ${ }^{5}$, PER J. NILSSON ${ }^{1}$ and HENRIK IVERSEN ${ }^{1}$ \\ ${ }^{1}$ Department of Molecular Medicine and Surgery, Karolinska Institutet, Division of Coloproctology, \\ Center for Digestive Diseases, Karolinska University Hospital, Stockholm, Sweden; \\ ${ }^{2}$ Department of Imaging and Physiology, Karolinska University Hospital, Stockholm, Sweden; \\ ${ }^{3}$ Department of Molecular Medicine and Surgery, Karolinska Institutet, \\ Department of Imaging and Physiology, Karolinska University Hospital, Stockholm, Sweden; \\ ${ }^{4}$ Department of Oncology-Pathology, Karolinska Institutet, Stockholm, Sweden; \\ ${ }^{5}$ Department of Immunology, Genetics and Pathology, Uppsala University, Uppsala, Sweden
}

\begin{abstract}
Background/Aim: For patients with locally recurrent rectal cancer (LRRC) extensive surgery is often the only curative option and patient selection is crucial. This study aimed to investigate whether magnetic resonance imaging (MRI) characteristics of the primary tumour can predict oncological outcome after surgery for locally recurrent rectal cancer (LRRC). Patients and Methods: All patients undergoing surgery for LRRC with a curative intent at the Karolinska University Hospital 2003-2013 were included. MRI examinations of the primary tumour were re-evaluated. Results: In total, 54 patients were included. A tumour volume decrease of $<70 \%$ after preoperative radiotherapy or chemoradiotherapy (C)RT for the primary tumour was correlated with a lower proportion of $R O$ resection of the LRRC (OR=0.07, 95\% $C I=0.01-0.84)$. No association between MRI characteristics of the primary tumour and prognosis after LRRC surgery was found. Conclusion: Long-term outcomes after surgery for LRRC were not significantly associated with MRI characteristics of the index tumour. However, factors associated with increased risk of RI resection of LRRC were identified.
\end{abstract}

Local recurrence rates after rectal cancer surgery have been substantially reduced following the introduction of total

This article is freely accessible online.

Correspondence to: Anders Elliot, MD, Ph.D., Division of Coloproctology, Center for Digestive Diseases, Anna Steckséns gata 30A, D2:05, Karolinska University Hospital, 17176 Stockholm, Sweden. Tel: +46736 332275, e-mail: anders.hansson-elliot@ sll.se

Key Words: Rectal cancer, local recurrence, MRI, preoperative treatment, oncological outcome. mesorectal excision (TME) surgery (1). Preoperative radiotherapy (RT) or chemoradiotherapy (CRT) for rectal cancer have further decreased the numbers of locally recurrent rectal cancer (LRRC) (2-4). Although improvement over time has occurred, patients undergoing surgery for LRRC have relatively poor survival outcomes, especially if an $\mathrm{R} 0$ resection is not accomplished (5-10).

Magnetic resonance imaging (MRI) has become standard for pretherapeutic staging and for the assessment of local tumour status after preoperative (C)RT in primary rectal cancer. Several tumour characteristics readily identifiable on pretherapeutic MRI of the primary tumour, predict for the later development of LRRC (11). Although with limited data available, a MRI-defined poor tumour regression grade (mrTRG) and a limited reduction of tumour volume on primary tumour MRI after preoperative (C)RT appear to correspond to a poor histopathological tumour response and are associated with inferior oncological outcomes (12-15).

Apart from patient factors such as advanced age and comorbidity, tumour factors such as disseminated disease and features of the LRRC on MRI play a key role for selection to surgery for LRRC (16). However, it is not fully explored if primary tumour characteristics may have a predictive value regarding long-term outcome after LRRC surgery. According to some recent reports, advanced histopathological $\mathrm{T}$ and $\mathrm{N}$ stage of the primary tumour corresponds to inferior prognosis after LRRC but there is limited data on the ability to predict long-term oncological outcomes after surgery for LRRC based on primary tumour MRI $(7,17)$.

Preoperative (C)RT in rectal cancer is associated with morbidity $(18,19)$. Furthermore, morbidity following surgery for LRRC is considerable $(6,20)$. Hence, all available information must be considered in treatment selection for both primary rectal cancer and LRRC to avoid over- and under-treatment. 
The aim was to investigate whether there are factors on pre-treatment and restaging MRI for primary rectal cancer of predictive importance regarding outcomes following surgery with a curative intent for LRRC. In addition effects of treatment selection for the primary tumour and outcomes with respect to the LRRC were explored.

\section{Patients and Methods}

Patients. Karolinska University Hospital is a tertiary referral centre for LRRC. All patients who underwent surgery for LRRC with a curative intent at the Karolinska University Hospital between January 2003 and December 2013 and had an MRI before surgery for the primary tumour were included. Information regarding age, sex, date and type of primary surgery, and preoperative and adjuvant treatment for the primary tumour was retrieved from patient records. For the LRRC, data collection included information on date and type of surgery, histopathology report, end of follow-up date, death and distant and/or local re-recurrence. For all patients, information was also retrieved concerning occurrence of and treatment for distant metastases. The study was approved by the regional Ethical Review Board at Karolinska Institutet (approval number: KI-00-135, 2008/1939-32, 2011/1481-32, 2012/1975-32, 2016/2487-32, 04576/2, 2009/1468-32, 2014/1573-32) (Stockholm, Sweden).

MRI re-evaluation. Pelvic MR examinations of the primary rectal cancer were retrieved for all patients. In patients in whom a restaging MRI had been performed after preoperative therapy, these examinations were also collected. All MR images were re-evaluated a first time by a body radiologist specialised in rectal cancer MRI (NB) and, if judged necessary by the first radiologist, a second time together with a second radiologist specialised in rectal cancer MRI (either LB or CS). When re-evaluating the primary tumour MRIs, the radiologists were blinded for the original MRI report. Re-evaluation of the pretherapeutic MRI included tumour level above anal verge, tumour volume, $\mathrm{mrT}$ stage, $\mathrm{mrN}$ stage, presence of suspicious lateral lymph nodes, extramural vascular invasion (mrEMVI) status and involvement of the mesorectal fascia (mrMRF). Lateral lymph nodes were defined as anatomically located along the internal iliac artery and in the obturator fossae. Morphological criteria on pretherapeutic T2-weighted images were used to define presence of lymph node metastases $(21,22)$. Restaging MR images were re-evaluated with respect to tumour volume and mrTRG. A 70\% decrease in tumour volume between the pre-treatment staging MRI and the restaging MRI was considered as a significant response based on previous studies $(13,23)$. mrTRG was assessed using a five-graded scale according to experiences from the MERCURY study group $(24,25)$. An mrTRG of 1, 2 and 3 represents a complete, near complete and moderate response, respectively, while an mrTRG of 4 and 5 a slight and no response with a predominance of tumour, respectively.

Preoperative treatment. After re-evaluation of the pre-treatment MRI before primary surgery, all primary tumours were classified as early, intermediate or locally advanced according to a previous publication and related to the selected preoperative treatment regimen in each patient (26). According to the Swedish guidelines, early, intermediate, and locally advanced tumours should be selected for no preoperative treatment, preoperative short-course RT and preoperative CRT, respectively (26).
Outcomes. The outcome measures included overall survival (OS), disease-free survival (DFS) and local re-recurrence. OS was defined as time from LRRC surgery to death from any cause and DFS as time from LRRC surgery to local or systemic re-recurrence or death from any cause.

Statistical analysis. For comparison of categorical variables the chisquare test was used. The Kaplan-Meier method was used to estimate rates of DFS, OS and local re-recurrence. Differences were assessed by the log-rank test. All $p$-values $<0.050$ were considered statistically significant. To identify risk factors for outcome measures, OS, DFS, and local re-recurrence after surgery for LRRC present at the time of primary surgery, univariable Cox regression analyses were used. Predictors for non-radical LRRC surgery were identified with univariable logistic regression analyses. Wald test was used to test for trends. Data were analysed using Stata ${ }^{\circledR}$ version 12.0 statistical software package (Stata Corp LP, College Station, Texas, USA).

\section{Results}

Patients. During the study period, a total of 76 patients underwent surgery for LRRC. Two patients were excluded as surgery was indicated by re-recurrence. Because of unavailability of MRI before primary surgery, another 20 patients were excluded, leaving 54 patients for analyses. Table I summarizes descriptive data on these 54 patients.

Primary tumour. Prior to surgery for the primary tumour, 42 (78\%) patients received preoperative (C)RT. Among those patients, 14 had direct surgery and 20 had a restaging MRI after preoperative therapy. In the remaining 8 patients, a restaging MRI was not available.

Of all patients, $15(28 \%)$ were treated with adjuvant chemotherapy for the primary tumour. Two patients had synchronous metastases at primary surgery and 9 patients were treated for metachronous metastases before LRRC surgery.

Of all primary tumours at re-evaluation, 10 (19\%) were classified as early, $14(26 \%)$ as intermediate and $30(56 \%)$ as locally advanced (Table II). Among the 30 patients with locally advanced tumours according to MRI, $20(67 \%)$ patients did not receive preoperative CRT. Out of these 20 patients, 12 were 65 years or younger. In total, preoperative treatment of a lower intensity than that recommended by the Swedish guidelines was delivered to $24(45 \%)$ patients.

Local recurrence. Median age at surgery for the LRRC was 65 years (range $=42-82$ ). The median time interval between primary surgery and LRRC surgery was 28 months (range=5-146). There were no in-hospital deaths. Among the 42 patients initially treated with preoperative (C)RT, reirradiation for the LRRC was given to $13(31 \%)$ patients. In the group of 12 patients previously non-irradiated, 6 patients received RT before LRRC surgery, 2 patients received intraoperative RT and 4 patients were never irradiated. Eight 
Table I. Clinical and radiological characteristics of the primary rectal cancer $(n=54)$.

\begin{tabular}{|c|c|}
\hline & $\mathrm{n}(\%)$ \\
\hline Age at primary surgery*, years & $63(41-82)$ \\
\hline$<65$ & $32(59)$ \\
\hline$\geq 65$ & $22(41)$ \\
\hline \multicolumn{2}{|l|}{ Gender } \\
\hline Male & $33(61)$ \\
\hline Female & $21(39)$ \\
\hline \multicolumn{2}{|l|}{ Tumour height, MRI (cm) } \\
\hline $0-5$ & $16(30)$ \\
\hline $6-10$ & $23(43)$ \\
\hline $10-15$ & $14(26)$ \\
\hline \multicolumn{2}{|l|}{ mrT stage } \\
\hline T0-T2 & $11(20)$ \\
\hline $\mathrm{T} 3$ & $23(43)$ \\
\hline $\mathrm{T} 4$ & $20(37)$ \\
\hline \multicolumn{2}{|l|}{$\mathrm{mrN}$ stage } \\
\hline No & $11(20)$ \\
\hline N1 & $26(48)$ \\
\hline $\mathrm{N} 2$ & $17(32)$ \\
\hline \multicolumn{2}{|l|}{ mrMRF status } \\
\hline Positive & $25(46)$ \\
\hline Negative & $29(54)$ \\
\hline \multicolumn{2}{|l|}{ mrEMVI } \\
\hline Yes & $28(52)$ \\
\hline No & $26(48)$ \\
\hline \multicolumn{2}{|l|}{ Involved lateral nodes, MRI } \\
\hline Yes & $13(24)$ \\
\hline No & $41(76)$ \\
\hline \multicolumn{2}{|l|}{ Tumour volume decrease, MRI** } \\
\hline$>70 \%$ & $10(50)$ \\
\hline$\leq 70 \%$ & $10(50)$ \\
\hline \multicolumn{2}{|l|}{ mrTRG** } \\
\hline $1-3$ & $14(70)$ \\
\hline $4-5$ & $6(30)$ \\
\hline
\end{tabular}

MRI, Magnetic resonance imaging; mr, MRI-defined; MRF, mesorectal fascia; EMVI, extramural vascular invasion; TRG, tumour regression grade. *Data presented as median (range); $* * n=20$.

patients received adjuvant chemotherapy after LRRC surgery and in 3 of those patients adjuvant chemotherapy had been delivered also after primary surgery.

Outcomes. R0 resection of the LRRC was achieved in 35 $(65 \%)$ patients and the remaining 19 patients had R1 resections. In Table III, the univariable logistic regression analyses of associations between radiological factors on pretreatment MRI of the primary tumour and R0 surgery of the LRRC are shown. No statistically significant associations could be detected except among the 20 patients with a available restaging MRI after preoperative (C)RT. In those patients in whom primary tumour volume decrease was less than $70 \%$ after (C)RT, R0 surgery for the LRRC was less likely to occur $(\mathrm{OR}=0.07,95 \% \mathrm{CI}=0.01-0.84)$. Also an
Table II. Pre-treatment stage according to magnetic resonance imaging (MRI) re-evaluation related to preoperative treatment of the primary rectal cancer $(n=54)$.

\begin{tabular}{|c|c|c|c|c|}
\hline \multirow[t]{2}{*}{ Tumour stage } & \multirow{2}{*}{$\begin{array}{c}\text { Total } \\
\mathrm{n}\end{array}$} & \multicolumn{3}{|c|}{ Preoperative treatment } \\
\hline & & No & RT & CRT \\
\hline Early & 10 & $4(40)$ & $6(60)$ & $0(0)$ \\
\hline Intermediate & 14 & $4(29)$ & $9(64)$ & $1(7)$ \\
\hline Locally advanced & 30 & $4(13)$ & $16(53)$ & $10(33)$ \\
\hline
\end{tabular}

RT, Radiotherapy; CRT, chemoradiotherapy. Values in parentheses are percentages.

mrTRG of 4-5 was associated with a decreased chance of R0 resection of the LRRC $(\mathrm{OR}=0.14,95 \% \mathrm{CI}=0.02-1.14)$, however without statistical significance.

The 3-year OS, DFS and local re-recurrence rate after LRRC surgery were $57 \%, 26 \%$ and $41 \%$, respectively. One patient had synchronous liver metastases at the time of LRRC surgery and was therefore excluded from the analyses of DFS. There were no statistically significant differences between women and men in terms of OS and DFS. R0 resection of the local recurrence was a strong predictor for 3-year OS (R0 66\% vs. R1 42\%, $p=0.005$ ) and DFS (R0 $31 \%$ vs. R1 7\%, $p=0.024)$. Patients 65 years or older had local re-recurrence to a lesser extent than younger patients $(\mathrm{HR}=0.30,95 \% \mathrm{CI}=0.10-0.91)$. Patients who received preoperative (C)RT for the primary tumour had inferior OS following LRRC surgery compared to those without primary preoperative treatment $(p=0.028)$. MRI characteristics of the primary tumour with potential predictive value for the LRRC surgery are displayed in Table IV. There were no statistically significant associations between pretherapeutic primary tumour MRI variables such as mrT stage, mrN stage, mrMRF or mrEMVI and OS, DFS or local re-recurrence in the univariable Cox regression analyses.

\section{Discussion}

In this retrospective study, 54 patients who underwent surgery for LRRC were analysed and possible associations between primary tumour MRI characteristics and surgical outcomes were investigated. The results showed that no characteristics of the pre-treatment MRI could predict outcomes, whereas an evident response to preoperative (C)RT in the primary tumour, measured as decreased tumour volume, was associated with an increased chance of obtaining an R0 resection of the LRRC. Although not statistically significant, the result on mrTRG pointed in the same direction. It is conceivable that biological properties of a tumour influencing response to neoadjuvant therapy may 
Table III. Univariable logistic regression analyses of association between MRI characteristics of the primary tumour and RO surgery of the locally recurrent rectal cancer $(n=54)$.

\begin{tabular}{|c|c|c|c|c|}
\hline & \multirow{2}{*}{$\begin{array}{c}\text { Total LRRC } \\
n\end{array}$} & \multicolumn{3}{|c|}{$\mathrm{R} 0$ resection } \\
\hline & & $\mathrm{n}(\%)$ & OR $(95 \% \mathrm{CI})$ & $p$-Value* \\
\hline Tumour height, MRI $(\mathrm{cm})^{* *}$ & & & & 0.653 \\
\hline $0-5$ & 16 & $12(75)$ & 1.00 & \\
\hline $6-10$ & 23 & $14(61)$ & $0.52(0.13-2.12)$ & \\
\hline $11-15$ & 14 & $9(64)$ & $0.60(0.12-2.89)$ & \\
\hline mrT stage & & & & 0.927 \\
\hline $\mathrm{T} 0-\mathrm{T} 2$ & 11 & $7(64)$ & 1.00 & \\
\hline T3-4 & 43 & $28(65)$ & $1.07(0.27-4.24)$ & \\
\hline mrN stage & & & & 0.168 \\
\hline No & 11 & $9(82)$ & 1.00 & \\
\hline N1-2 & 43 & $26(60)$ & $0.34(0.07-1.77)$ & \\
\hline mrEMVI & & & & 0.290 \\
\hline No & 26 & $15(58)$ & 1.00 & \\
\hline Yes & 28 & $20(71)$ & $1.83(0.59-5.68)$ & \\
\hline mrMRF status & & & & 0.907 \\
\hline Positive & 29 & $19(66)$ & 1.00 & \\
\hline Negative & 25 & $16(64)$ & $0.94(0.31-2.87)$ & \\
\hline mrTRG*** & & & & \\
\hline $0-3$ & 14 & $11(79)$ & 1.00 & \\
\hline $4-5$ & 6 & $2(33)$ & $0.14(0.02-1.14)$ & \\
\hline Tumour volume decrease, $\mathrm{MRI}^{* * *}$ & & & & 0.015 \\
\hline$>70 \%$ & 10 & $9(90)$ & 1.00 & \\
\hline$\leq 70 \%$ & 10 & $4(40)$ & $0.07(0.01-0.84)$ & \\
\hline
\end{tabular}

LRRC, Locally recurrent rectal cancer; MRI, magnetic resonance imaging; mr, MRI-defined; MRF, mesorectal fascia; EMVI, extramural vascular invasion; TRG, tumour regression grade. $*$ Wald test. $* *$ One missing patient because of primary tumour undetectable on MRI. ***n=20.

also have an impact on the LRRC and the possibilities to successfully treat the LRRC. The experience of pre- or postoperative re-irradiation is limited; however, some recent investigations demonstrated that re-irradiation of LRRC, possibly with the addition of induction chemotherapy, resulted in enhanced survival and local control, acceptable morbidity, as well as a possibility of complete pathological response $(27,28)$. There are indications that primary tumour response to neoadjuvant (C)RT predicts outcomes after rectal cancer surgery and possibly the response to preoperative (C)RT for the primary tumour could be helpful to guide in the selection of treatment of the LRRC (29).

In this series of patients, it appears as if a substantial proportion of patients were undertreated with respect to the primary tumour, since $2 / 3$ of patients with a locally advanced tumour did not receive CRT as recommended. Previous studies have shown that advanced age and comorbidity are important factors and also that the selection strategy may have an impact on outcomes following treatment of primary rectal cancer $(30,31)$. In the present study, patients were relatively young (median age 63 years) and subsequently were considered fit for surgery of a LRRC. Therefore, neither advanced age nor significant co-morbidity appear as reasonable explanations for this under-treatment. Since the patients in this study were included from 2003, the recommendations of preoperative treatment have changed throughout the study period and this could partly explain the under-treatment. Also, previous pelvic irradiation as a reason for omittance of preoperative RT of the rectal cancer was not possible to control for. However, given the relatively young median age among the studied patients, this explanation seems less likely.

The finding that patients who had received primarily neoadjuvant (C)RT had an inferior OS after LRRC surgery should not be interpreted so that (C)RT should be avoided when indicated; rather, tumours that are initially more aggressive produce more aggressive recurrences, resulting in poorer survival rates. Treatment for LRRC is demanding and results, although gradually improving, are still poor in comparison with treatment for primary rectal cancer (32). Thus, a main objective in rectal cancer treatment should be to avoid a LRRC and obtain sustained local control. The present series indicate that there may be room for improved treatment selection in primary rectal cancer management and, theoretically, some of the LRRCs in this series may have been avoided had (C)RT been given. 
Table IV. Univariable Cox regression analyses of association between MRI characteristics of the primary tumour and outcome after surgery for locally recurrent rectal cancer $(n=54)$.

\begin{tabular}{|c|c|c|c|}
\hline & $\begin{array}{l}\text { Overall survival } \\
\text { HR }(95 \% \mathrm{CI})\end{array}$ & $\begin{array}{c}\text { Disease-free survival* } \\
\text { HR }(95 \% \mathrm{CI})\end{array}$ & $\begin{array}{c}\text { Local re-recurrence } \\
\text { HR }(95 \% \mathrm{CI})\end{array}$ \\
\hline \multicolumn{4}{|c|}{ Tumour height, MRI $(\mathrm{cm})^{* *}$} \\
\hline $0-5$ & 1.00 & 1.00 & 1.00 \\
\hline $6-10$ & $0.78(0.35-1.71)$ & $1.10(0.54-2.24)$ & $0.77(0.28-2.14)$ \\
\hline $10-15$ & $1.31(0.55-3.15)$ & $1.25(0.54-2.87)$ & $0.85(0.25-2.92)$ \\
\hline \multicolumn{4}{|l|}{ mrT stage } \\
\hline T0-2 & 1.00 & 1.00 & 1.00 \\
\hline $\mathrm{T} 3-4$ & $1.21(0.53-2.77)$ & $1.32(0.63-2.76)$ & $1.77(0.52-6.06)$ \\
\hline \multicolumn{4}{|l|}{$\mathrm{mrN}$ stage } \\
\hline No & 1.00 & 1.00 & 1.00 \\
\hline N1-2 & $1.53(0.64-3.69)$ & $1.70(0.79-3.67)$ & $3.02(0.70-13.05)$ \\
\hline \multicolumn{4}{|l|}{ mrMRF status } \\
\hline Positive & 1.00 & 1.00 & 1.00 \\
\hline Negative & $1.20(0.63-2.30)$ & $1.52(0.83-2.77)$ & $1.26(0.52-3.02)$ \\
\hline \multicolumn{4}{|l|}{ mrEMVI } \\
\hline No & 1.00 & 1.00 & 1.00 \\
\hline Yes & $1.21(0.62-2.35)$ & $1.18(0.65-2.15)$ & $1.69(0.69-4.15)$ \\
\hline \multicolumn{4}{|c|}{ Involved lateral nodes, MRI } \\
\hline No & 1.00 & 1.00 & 1.00 \\
\hline Yes & $1.20(0.56-2.57)$ & $1.33(0.67-2.65)$ & $1.54(0.59-4.02)$ \\
\hline \multicolumn{4}{|l|}{ mrTRG*** } \\
\hline $0-3$ & 1.00 & 1.00 & 1.00 \\
\hline $4-5$ & $2.20(0.71-6.84)$ & $2.05(0.74-5.72)$ & $2.01(0.58-6.98)$ \\
\hline \multicolumn{4}{|c|}{ Tumour volume decrease, MRI*** } \\
\hline$>70 \%$ & 1.00 & 1.00 & 1.00 \\
\hline$\leq 70 \%$ & $1.07(0.39-2.96)$ & $0.76(0.30-1.93)$ & $1.69(0.49-5.80)$ \\
\hline \multicolumn{4}{|c|}{ Preoperative tumour stage, MRI } \\
\hline Early & 1.00 & 1.00 & 1.00 \\
\hline Intermediate & $1.36(0.48-3.84)$ & $1.23(0.49-3.14)$ & $2.46(0.48-12.70)$ \\
\hline Locally advanced & $1.32(0.64-3.84)$ & $1.48(0.66-3.29)$ & $2.70(0.61-11.98)$ \\
\hline
\end{tabular}

MRI, Magnetic resonance imaging; mr, MRI-defined; MRF, mesorectal fascia; EMVI, extramural vascular invasion; TRG, tumour regression grade. *One patient excluded because of synchronous metastases by primary surgery. **One missing patient because of primary tumour undetectable on MRI. $* * * n=20$.

The association between age and local re-recurrence where older patients had better local control after LRRC surgery is possibly due to the fact that younger patients are being selected for curatively intended surgery for LRRC to a higher extent, also with more advanced tumours, than older patients.

There are several limitations that need to be addressed. The retrospective approach and the small study population create obvious restrictions. Only 20 of 42 patients treated with preoperative (C)RT could be assessed regarding mrTRG and tumour volume decrease. Local recurrence rates in rectal cancer are low today (33). Furthermore, far from all patients with a LRRC can be offered surgery with a curative intent; therefore, few patients are available for inclusion into clinical investigations. Prospective studies on rare conditions are difficult to perform and implicate a long study inclusion period. An international registry of LRRCpatients with prospectively collected data could possibly improve the possibilities to design investigations with sufficient quality. Secondly, the LRRC surgery of the patients was carried out over a relatively long time span. Treatment of both the primary tumour and the LRRC has evolved over time, especially regarding preoperative (C)RT, and this could naturally have an impact on prognosis. Thirdly, many factors other than the features of the primary tumour may influence the outcome after LRRC surgery. There is an obvious risk of confounding because of differences in, for example, comorbidity, oncological treatment before and after LRRC and the histopathological stage of the primary tumour and LRRC.

In conclusion, it is well established that preoperative (C)RT for rectal cancer reduces the risk of LRRC. Still, among the patients who undergo surgery for LRRC, this study revealed that the primary tumour may have been under-treated in a substantial proportion of the patients. However, prediction of long-term outcome after LRRC surgery based on the 
radiological features on the pretherapeutic MRI for the primary tumour was not possible. Improved decisions on both surgical and preoperative oncological therapy in rectal cancer patients can reduce rates of LRRC and possibly also improve survival, as well. Multicentre investigations and international prospectively collected registries are warranted to increase knowledge about the management and prevention of LRRC in rectal cancer.

\section{Conflicts of Interest}

The Authors declare that they have no conflicts of interest in regard to this study.

\section{Authors' Contributions}

Study concepts and design: All Authors; Data acquisition: AE, BO, CS, LB and HI; Data analysis and interpretation: AE, LB, HJ, BG, $\mathrm{PN}$ and HI; Statistical analysis: AE and HJ; Manuscript preparation: $\mathrm{AE}$ and HI; Manuscript editing and approval of the final article: All Authors.

\section{Acknowledgements}

This project was supported by the Swedish Cancer Society, the Stockholm Cancer Society and the Regional agreement on medical training and clinical research (ALF) between the Stockholm County Council and Karolinska Institutet.

\section{References}

1 Heald R and Ryall R: Recurrence and survival after total mesorectal excision for rectal cancer. The Lancet 327(8496): 1479-1482, 2019. DOI: 10.1016/s0140-6736(86)91510-2

2 van Gijn W, Marijnen CA, Nagtegaal ID, Kranenbarg EM, Putter H, Wiggers T, Rutten HJ, Påhlman L, Glimelius B, van de Velde CJ and Dutch Colorectal Cancer Group: Preoperative radiotherapy combined with total mesorectal excision for resectable rectal cancer: 12-year follow-up of the multicentre, randomised controlled TME trial. Lancet Oncol 12(6): 575-582, 2011. PMID: 21596621. DOI: 10.1016/S1470-2045(11)70097-3

3 Sebag-Montefiore D, Stephens RJ, Steele R, Monson J, Grieve R, Khanna S, Quirke P, Couture J, de Metz C, Myint AS, Bessell E, Griffiths G, Thompson LC and Parmar M: Preoperative radiotherapy versus selective postoperative chemoradiotherapy in patients with rectal cancer (MRC CR07 and NCIC-CTG C016): a multicentre, randomised trial. Lancet 373(9666): 811-820, 2009. PMID: 19269519. DOI: 10.1016/S0140-6736(09)60484-0

4 Swedish Rectal Cancer Trial., Cedermark B, Dahlberg M, Glimelius B, Påhlman L, Rutqvist LE and Wilking N: Improved survival with preoperative radiotherapy in resectable rectal cancer. N Engl J Med 336(14): 980-987, 1997. PMID: 9091798. DOI: $10.1056 /$ NEJM199704033361402

5 Bhangu A, Ali SM, Darzi A, Brown G and Tekkis P: Meta-analysis of survival based on resection margin status following surgery for recurrent rectal cancer. Colorectal Dis 14(12): 1457-1466, 2012. PMID: 22356246. DOI: 10.1111/j.1463-1318.2012.03005.x
6 Bosman SJ, Vermeer TA, Dudink RL, de Hingh IH, Nieuwenhuijzen GA and Rutten HJ: Abdominosacral resection: long-term outcome in 86 patients with locally advanced or locally recurrent rectal cancer. Eur J Surg Oncol 40(6): 699-705, 2014. PMID: 24679359. DOI: 10.1016/j.ejso.2014.02.233

7 Du P, Burke JP, Khoury W, Lavery IC, Kiran RP, Remzi FH and Dietz DW: Factors associated with the location of local rectal cancer recurrence and predictors of survival. Int J Colorectal Dis 31(4): 825-832, 2016. PMID: 26861707. DOI: 10.1007/s00384016-2526-5

8 Harris CA, Solomon MJ, Heriot AG, Sagar PM, Tekkis PP, Dixon L, Pascoe R, Dobbs BR, Frampton CM, Harji DP, Kontovounisios C, Austin KK, Koh CE, Lee PJ, Lynch AC, Warrier SK and Frizelle FA: The outcomes and patterns of treatment failure after surgery for locally recurrent rectal cancer. Ann Surg 264(2): 323-329, 2016. PMID: 26692078. DOI: 10.1097/SLA.0000000000001524

9 Nielsen M, Rasmussen P, Pedersen B, Hagemann-Madsen R, Lindegaard J and Laurberg S: Early and late outcomes of surgery for locally recurrent rectal cancer: A prospective 10-year study in the total mesorectal excision era. Ann Surg Oncol 22(8): 26772684, 2015. PMID: 25564165. DOI: 10.1245/s 10434-014-4317-y

10 Iversen H, Martling A, Johansson H, Nilsson PJ and Holm T: Pelvic local recurrence from colorectal cancer: surgical challenge with changing preconditions. Colorectal Dis 20(5): 399-406, 2018. PMID: 29161761. DOI: 10.1111/codi.13966

11 Glynne-Jones R, Tan D and Goh V: Pelvic MRI for guiding treatment decisions in rectal cancer. Oncology (Williston Park) 28(8): 667-677, 2014. PMID: 25140623.

12 Patel UB, Taylor F, Blomqvist L, George C, Evans H, Tekkis P, Quirke P, Sebag-Montefiore D, Moran B, Heald R, Guthrie A, Bees N, Swift I, Pennert K and Brown G: Magnetic resonance imaging-detected tumor response for locally advanced rectal cancer predicts survival outcomes: MERCURY experience. J Clin Oncol 29(28): 3753-3760, 2011. PMID: 21876084. DOI: 10.1200/JCO.2011.34.9068

13 Nougaret S, Rouanet P, Molinari N, Pierredon MA, Bibeau F, Azria D, Lemanski C, Assenat E, Duffour J, Ychou M, Reinhold $\mathrm{C}$ and Gallix B: MR volumetric measurement of low rectal cancer helps predict tumor response and outcome after combined chemotherapy and radiation therapy. Radiology 263(2): 409-418, 2012. PMID: 22438364. DOI: 10.1148/radiol.12111263

14 Martens MH, van Heeswijk MM, van den Broek JJ, Rao SX, Vandecaveye V, Vliegen RA, Schreurs WH, Beets GL, Lambregts DM and Beets-Tan RG: Prospective, multicenter validation study of magnetic resonance volumetry for response assessment after preoperative chemoradiation in rectal cancer: can the results in the literature be reproduced? Int J Radiat Oncol Biol Phys 93(5): 1005-1014, 2015. PMID: 26581139. DOI: 10.1016/j.ijrobp.2015.09.008

15 Sclafani F, Brown G, Cunningham D, Wotherspoon A, Mendes LST, Balyasnikova S, Evans J, Peckitt C, Begum R, Tait D, Tabernero J, Glimelius B, Roselló S, Thomas J, Oates J and Chau I: Comparison between MRI and pathology in the assessment of tumour regression grade in rectal cancer. Br J Cancer 117(10): 1478-1485, 2017. PMID: 28934761. DOI: 10.1038/bjc. 2017.320

16 Brown WE, Koh CE, Badgery-Parker T and Solomon MJ: Validation of MRI and surgical decision making to predict a complete resection in pelvic exenteration for recurrent rectal 
cancer. Dis Colon Rectum 60(2): 144-151, 2017. PMID: 28059910. DOI: 10.1097/DCR.0000000000000766

17 Selvaggi F, Fucini C, Pellino G, Sciaudone G, Maretto I, Mondi I, Bartolini N, Caminati F and Pucciarelli S: Outcome and prognostic factors of local recurrent rectal cancer: a pooled analysis of 150 patients. Tech Coloproctol 19(3): 135-144, 2015. PMID: 25384359. DOI: 10.1007/s10151-014-1241-x

18 Birgisson H, Påhlman L, Gunnarsson U and Glimelius B: Late adverse effects of radiation therapy for rectal cancer - a systematic overview. Acta Oncol 46(4): 504-516, 2007. PMID: 17497318. DOI: $10.1080 / 02841860701348670$

19 Marijnen CA, Kapiteijn E, van de Velde CJ, Martijn H, Steup WH, Wiggers T, Kranenbarg EK, Leer JW and Cooperative Investigators of the Dutch Colorectal Cancer Group: Acute side effects and complications after short-term preoperative radiotherapy combined with total mesorectal excision in primary rectal cancer: report of a multicenter randomized trial. J Clin Oncol 20(3): 817-825, 2002. PMID: 11821466. DOI: 10.1200/JCO.2002.20.3.817

20 Bhangu A, Brown G, Akmal M and Tekkis P: Outcome of abdominosacral resection for locally advanced primary and recurrent rectal cancer. Br J Surg 99(10): 1453-1461, 2012. PMID: 22961529. DOI: 10.1002/bjs.8881

21 Brown G, Richards CJ, Bourne MW, Newcombe RG, Radcliffe AG, Dallimore NS and Williams GT: Morphologic predictors of lymph node status in rectal cancer with use of high-spatialresolution MR imaging with histopathologic comparison. Radiology 227(2): 371-377, 2003. PMID: 12732695. DOI: 10.1148/radiol.2272011747

22 MERCURY Study Group, Shihab OC, Taylor F, Bees N, Blake H, Jeyadevan N, Bleehen R, Blomqvist L, Creagh M, George C, Guthrie A, Massouh H, Peppercorn D, Moran BJ, Heald RJ, Quirke P, Tekkis P and Brown G: Relevance of magnetic resonance imaging-detected pelvic sidewall lymph node involvement in rectal cancer. Br J Surg 98(12): 1798-1804, 2011. PMID: 21928408. DOI: 10.1002/bjs.7662

23 Barbaro B, Fiorucci C, Tebala C, Valentini V, Gambacorta MA, Vecchio FM, Rizzo G, Coco C, Crucitti A, Ratto C and Bonomo L: Locally advanced rectal cancer: MR imaging in prediction of response after preoperative chemotherapy and radiation therapy. Radiology 250(3): 730-739, 2009. PMID: 19244043. DOI: 10.1148/radiol.2503080310

24 Shihab OC, Taylor F, Salerno G, Heald RJ, Quirke P, Moran BJ and Brown G: MRI predictive factors for long-term outcomes of low rectal tumours. Ann Surg Oncol 18(12): 3278-3284, 2011. PMID: 21590453. DOI: 10.1245/s10434-011-1776-2
25 Patel UB, Blomqvist LK, Taylor F, George C, Guthrie A, Bees $\mathrm{N}$ and Brown G: MRI after treatment of locally advanced rectal cancer: how to report tumor response - the MERCURY experience. AJR Am J Roentgenol 199(4): W486-W495, 2012. PMID: 22997398. DOI: 10.2214/AJR.11.8210

26 Blomqvist L and Glimelius B: The 'good', the 'bad', and the 'ugly' rectal cancers. Acta Oncol 47(1): 5-8, 2008. PMID: 18097776. DOI: $10.1080 / 02841860701802585$

27 van der Meij W, Rombouts AJ, Rütten H, Bremers AJ and de Wilt JH: Treatment of locally recurrent rectal carcinoma in previously (chemo)irradiated patients: A review. Dis Colon Rectum 59(2): 148-156, 2016. PMID: 26734974. DOI: 10.1097/ DCR.0000000000000547

28 Voogt ELK, van Zoggel DMGI, Kusters M, Nieuwenhuijzen GAP, Bloemen JG, Peulen HMU, Creemers GJM, van Lijnschoten G, Nederend J, Roef MJ, Burger JWA and Rutten HJT: Improved outcomes for responders after treatment with induction chemotherapy and chemo(re)irradiation for locally recurrent rectal cancer. Ann Surg Oncol 27(9): 3503-3513, 2020. PMID: 32193717. DOI: 10.1245/s10434-020-08362-4

29 Nougaret S, Castan F, de Forges H, Vargas HA, Gallix B, Gourgou S, Rouanet P and GRECCAR Study Group: Early MRI predictors of disease-free survival in locally advanced rectal cancer from the GRECCAR 4 trial. Br J Surg 106(11): 15301541, 2019. PMID: 31436325. DOI: 10.1002/bjs.11233

30 Elliot AH, Martling A, Glimelius B, Nordenvall C, Johansson H and Nilsson PJ: Preoperative treatment selection in rectal cancer: A population-based cohort study. Eur J Surg Oncol 40(12): 17821788, 2014. PMID: 25260598. DOI: 10.1016/j.ejso.2014.08.481

31 Elliot AH, Martling A, Glimelius B, Johansson H and Nilsson PJ: Impact of pre-treatment patient-related selection parameters on outcome in rectal cancer. Eur J Surg Oncol 42(11): 16671673, 2016. PMID: 27318529. DOI: 10.1016/j.ejso.2016.05.020

32 Bhangu A, Ali SM, Brown G, Nicholls RJ and Tekkis P: Indications and outcome of pelvic exenteration for locally advanced primary and recurrent rectal cancer. Ann Surg 259(2): 315-322, 2014. PMID: 23478530. DOI: 10.1097/SLA.0b013e31828a0d22

33 Glimelius B, Myklebust TÅ, Lundqvist K, Wibe A and Guren MG: Two countries - Two treatment strategies for rectal cancer. Radiother Oncol 121(3): 357-363, 2016. PMID: 27887734. DOI: 10.1016/j.radonc.2016.11.010

Received December 31, 2020 Revised March 1, 2021 Accepted April 12, 2021 